\title{
The Hausdorff Dimension of the Penrose Universe
}

\author{
L. Marek-Crnjac \\ Department of Mathematics, Technical School Center of Maribor, 2000 Maribor, Slovenia \\ Correspondence should be addressed to L. Marek-Crnjac, leila.marek@guest.arnes.si
}

Received 27 April 2011; Accepted 17 July 2011

Academic Editor: Leonardo Golubovic

Copyright (C) 2011 L. Marek-Crnjac. This is an open access article distributed under the Creative Commons Attribution License, which permits unrestricted use, distribution, and reproduction in any medium, provided the original work is properly cited.

Penrose fractal tiling is one of the simplest generic examples for a noncommutative space. In the present work, we determine the Hausdorff dimension corresponding to a four-dimensional analogue of the so-called Penrose Universe and show how it could be used in resolving various fundamental problems in high energy physics and cosmology.

\section{Introduction}

As explained in detail in Connes' [1], Penrose fractal tiling constitutes mathematically a quotient space $X$. Using this fact A. Connes following earlier work due to von Neumann deduced a dimensional function which we generalize to a simple formula function linking both the Menger-Urysohn topological dimension and the corresponding Hausdorff dimension.

The present work is subdivided into three main parts. First, we show an explicit application and generalization of the Connes' dimensional function. Second, we derive the Hausdorff dimension of the Hilbert space which $X$ represents. Finally, we show the relevance of these results in high energy physics and cosmology.

\section{The Dimensional Function and the Hilbert Space}

Let us start from the Connes' dimensional function for the Penrose universe [1]:

$$
D(a, b)=a+b \phi ; \quad \text { where } a, b \in \mathcal{Z}, \phi=\frac{\sqrt{5}-1}{2} .
$$

Writing $D_{n}\left(a_{n}, b_{n}\right)$ and using the Fibonacci sequence, it is easy to see that, starting from the seed $D_{0}=D(0,1)$ and $D_{1}=D(1,0)$, we obtain the following dimensional hierarchy:

$$
\begin{aligned}
& D_{0}(0,1)=0+\phi=\phi \\
& D_{1}(1,0)=1+(0) \phi=1, \\
& D_{2}(0+1,1+0)=1+\phi=\frac{1}{\phi}, \\
& D_{3}(1+1,0+1)=2+\phi=\left(\frac{1}{\phi}\right)^{2}, \\
& D_{4}(1+2,1+1)=3+2 \phi=\left(\frac{1}{\phi}\right)^{3}, \\
& D_{5}(2+3,1+2)=5+3 \phi=\left(\frac{1}{\phi}\right)^{4}, \\
& D_{n}\left(a_{n}, b_{n}\right)=\left(a_{n-1}+a_{n-2}\right)+\left(b_{n-1}+b_{n-2}\right) \phi=\left(\frac{1}{\phi}\right)^{n-1} .
\end{aligned}
$$

By complete induction, one finds

$$
D_{n}=\left(\frac{1}{\phi}\right)^{n-1}
$$

We obtain an exceptional Fibonacci sequence $F_{\phi}(n)$ :

$$
\{\phi, 1,1+\phi, 2+\phi, 3+2 \phi, 5+3 \phi, \ldots\}
$$


The classical Fibonacci sequence $F_{n}$ is defined by the recurrence relation

$$
F_{n+1}=F_{n}+F_{n-1}, \quad n \geq 1,
$$

where $F_{0}=0, F_{1}=1$, and $F_{2}=1$. The first few Fibonacci numbers of the classical Fibonacci sequence are given $\{0,1,1,2,3,5,8,13,21,34,55,89,144,233, \ldots\}$.

The $n$th Fibonacci number is given by the formula which is called the Binet form, named after Jaques Binet,

$$
F_{n}=\frac{\left(\phi^{-1}\right)^{n}-(-\phi)^{n}}{\phi^{-1}+\phi}
$$

where $\phi^{-1}$ and $-\phi$ are the solutions of the quadratic equation $x^{2}=x+1$ :

$$
x_{1}=\frac{\sqrt{5}+1}{2}=\frac{1}{\phi}, \quad x_{2}=\frac{1-\sqrt{5}}{2}=-\phi .
$$

The Binet form of the $n$th Fibonacci number of the $F_{\phi}(n)$ sequence can be expressed similar to the classical Fibonacci sequence:

$$
\begin{aligned}
& F_{\phi}(n)=\frac{\left(\phi^{-1}\right)^{n}-(-\phi)^{n}}{\phi^{-1}+\phi}+\frac{\left(\phi^{-1}\right)^{n-1}-(-\phi)^{n-1}}{\phi^{-1}+\phi} \phi, \quad n \geq 0, \\
& F_{\phi}(n)=\frac{1}{\phi^{-1}+\phi}\left(\left(\phi^{-1}\right)^{n}-(-\phi)^{n}\right. \\
& \left.+\left(\left(\phi^{-1}\right)^{n-1} \phi-(-\phi)^{n-1} \phi\right)\right) \\
& F_{\phi}(n)=\frac{1}{\phi^{-1}+\phi}\left(\left(\phi^{-1}\right)^{n}+\left(\phi^{-1}\right)^{n-1} \phi\right. \\
& \left.-(-\phi)^{n}-(-\phi)^{n-1} \phi\right) \\
& F_{\phi}(n)=\frac{\phi}{1+\phi^{2}}\left(\left(\phi^{-1}\right)^{n}\left(1+\phi^{2}\right)-(-\phi)^{n}\left(1+(-\phi)^{-1} \phi\right)\right) \text {, } \\
& F_{\phi}(n)=\left(\phi^{-1}\right)^{n-1} \text {. }
\end{aligned}
$$

The Fibonacci sequence $F_{\phi}(n)$ can be presented as an infinite geometric sequence:

$$
\begin{gathered}
\{\phi, 1,1+\phi, 2+\phi, 3+2 \phi, 5+3 \phi, \ldots\} \\
=\left\{\phi, \frac{1}{\phi^{0}}, \frac{1}{\phi^{1}}, \frac{1}{\phi^{2}}, \frac{1}{\phi^{3}}, \ldots\right\} .
\end{gathered}
$$

The Golden Section principle that connects the adjacent powers of the golden mean is seen from the infinite geometric sequence.

The formula for the $n$th Fibonacci number is clearly identical to the bijection formula of E-infinity algebra and rings, namely $[2,3]$,

$$
d_{c}^{(n)}=\left(\frac{1}{\phi}\right)^{n-1}
$$

Here, $n$ is the Menger-Urysohn topological dimension which should not be confused with the embedding dimension and $d_{c}^{(n)}$ is the Hausdorff dimension whose topological dimension is $n$. To see that this extends in a simple fashion $D\left(a_{n}, b_{n}\right)$ to negative dimensions [4], we set $n=-1$ and find that the empty set is structured and possesses a finite Hausdorff dimension equal to $\phi^{2}$ because

$$
d_{c}^{(-1)}=\left(\frac{1}{\phi}\right)^{-1-1}=\left(\frac{1}{\phi}\right)^{-2}=\phi^{2} .
$$

Now, we claim that $X$ is effectively a random Hilbert space and is four dimensional topologically speaking while the Hausdorff dimension is given by [5-7]

$$
\operatorname{dim} X_{(H)}=\frac{\operatorname{dim} \text { zeroset } \oplus \operatorname{dim} \text { emptyset }}{\operatorname{dim} \text { zeroset } \otimes \operatorname{dim} \text { emptyset }} .
$$

The zero set is given by

$$
d_{c}^{(0)}=\left(\frac{1}{\phi}\right)^{0-1}=\left(\frac{1}{\phi}\right)^{-1}=\phi .
$$

One finds

$$
\operatorname{dim} X_{(H)}=\frac{\phi+\phi^{2}}{\phi \phi^{2}}=\frac{1}{\phi^{3}}=4+\phi^{3}=4.236067977 .
$$

In an analogous manner, we see that the topological dimension is given by

$$
\operatorname{dim} X_{(H)}=\frac{1+(-1)}{0(-1)}=\infty .
$$

In other words, although $X$ has a finite average topological dimension equal to 4 , its formal topological dimension is infinity so that it is a real infinite-dimensional Hilbert space. Thus, the fact that

$$
d_{c}^{(4)}=\left(\frac{1}{\phi}\right)^{4-1}=\left(\frac{1}{\phi}\right)^{3}=4+\phi^{3}
$$

does not contradict the fact that the formal dimension is $\infty$ because 4 is an expectation value of the topological dimension in exactly the same manner that $4+\phi^{3}$ is the expectation value of the Hausdorff dimension. This fact is easily grasped when remembering that a random Cantor set has, according to Mauldin-Williams theorem, a Hausdorff dimension equal to $\phi$. Consequently, building a space $X$ from the union and the intersection of infinitely many such random Cantor sets gives us a hierarchal infinite-dimensional Cantor set with an average Hausdorff dimension given by the centre of gravity theorem [8-10]:

$$
\langle n\rangle=\langle d\rangle=\frac{\sum_{0}^{\infty} n^{2} \phi^{n}}{\sum_{0}^{\infty} n \phi^{n}}=\frac{1+\phi}{1-\phi}=4+\phi^{3}
$$

exactly as anticipated.

In fact it is well known from the work of Jones and Sunder's [11] and Connes' that the Hilbert space $L^{2}(N)$ is 
enclosed in $L^{2}(M)$ which has a dimension $\operatorname{dim}_{N}\left(L^{2}(M)\right)$ related to Jones' index $[M: N]$ of the subfactor $N \subseteq M$ by $[1,12]$

$$
\text { Index }[M: N]=\operatorname{dim}_{N}\left(L^{2}(M)\right) \geq 1 .
$$

It was shown in the E-infinity theory that $[1,12]$

$$
\operatorname{dim}_{N}\left(L^{2}(M)\right)=4+\phi^{3}
$$

as well as

$$
d(\infty)=4 .
$$

This clearly shows that $X$ is a Hilbert space as claimed. This very special Hilbert space is fixed by three and not only one dimension. It is formally infinite dimensional. Secondly, it has a finite average topological dimension equal to 4 . Thirdly, it has a finite average Hausdorff dimension equal to $4+\phi^{3}=$ 4.23606797. The infinite hierarchal nature of $X$ is reflected in the continued fraction expansion [13]:

$$
\begin{aligned}
\operatorname{dim} X_{(H)} & =\left(\frac{1}{\phi}\right)^{3}=4+\phi^{3} \\
& =(4)+(\overline{4}), \\
4+\phi^{3} & =4+\frac{1}{4+\frac{1}{4+\frac{1}{4+\ldots}}} .
\end{aligned}
$$

\section{Relevance in High Energy Physics}

With $\operatorname{dim} X_{(H)}$ at hand we can obtain a plethora of results in high energy physics by applying a simple intersection theory. As an example, we consider determining the value of the inverse electromagnetic fine structure constant $\bar{\alpha}_{o} \cong 137$. To do that, we generalize the two-dimensional Penrose tiling with $\operatorname{dim} X_{(H)}=4+\phi^{3}$ to a holographic boundary for a compact E-infinity manifold obtained from the fuzzy version of $E 8 E 8$ exceptional Lie symmetry groups for which we have [3]

$$
\begin{aligned}
\operatorname{dim} E 8 E 8 & =496-k^{2} \\
& =496-\left(\phi^{3}\left(1-\phi^{3}\right)\right)^{2} \\
& =495.9674775 \simeq 496,
\end{aligned}
$$

where $k=\phi^{3}\left(1-\phi^{3}\right)$.

Thus, intersecting $\operatorname{dim} X_{(H)}$ with the Narain lattice of superstring compactification with its well-known 80 dimensions gives

$$
\begin{aligned}
\operatorname{dim} \text { holographic boundary } & =\left(4+\phi^{3}\right)(80) \\
& =336+16 k \\
& \simeq 339,
\end{aligned}
$$

where 336 are the dimensions of $\operatorname{SL}(2,7)$ Lie group and the $16 k$ comes from the compactification. Noting that the number of symmetries of pure Einsteinium gravity in eight dimensions is exactly equal to 20 , then we can write the fundamental equation of the E-infinity theory as follows:

$$
\begin{aligned}
\text { Bulk }= & \text { holographic boundary }+ \text { gravity } \\
& + \text { electromagnetism. }
\end{aligned}
$$

That means

$$
\operatorname{dim}(E 8 E 8)=[S L(2,7)+16 k]+20+\bar{\alpha}_{o}
$$

Inserting and solving for $\bar{\alpha}_{o}$, one finds

$$
\bar{\alpha}_{o} \cong 496-(339+20)=137
$$

which is the integer approximation of the well-known experimental value. It should be noted that the 339 is the number of gluons-like states at ultra-high energy.

\section{Cosmology}

The vital role of the most irrational number, namely, the golden mean in the KAM theorem, is well understood and used extensively in the theory of quantum chaos. A similar role is played by the golden mean in the VAK [3] as well as the theory of vague torus [14] which links classical chaos and quantum mechanics. In some sense, the irrationality of $\phi$ is a substitute for the lack of friction in quantum Hamiltonian dynamics and gives elementary particles their stability. Researchers in cosmology have suspected for a long time that the Kirkwood gaps $[14,15]$ in the asteroids belt are due to the resonance instability connected to the lack of irrationality in the corresponding winding numbers and thus the lack of stability of the concerned orbits. We can transfer the same cosmological argument to high energy physics and claim that the hierarchal problem is due to similar stability considerations. It is reasoned in the E-infinity theory that the $\bar{\alpha}_{o}$ and $\phi$ quantization observed in the mass spectrum of elementary and composite particles is due to $\phi$ stability of $\operatorname{KAM}[14,15]$.

\section{Conclusion}

Penrose tiling is an incredibly rich but basically simple structure which is quantum mechanical in its very essence. It is a generic example of noncommutative geometry and the E-infinity theory. Penrose tiling may be generalized to 4 dimensions. The Hausdorff dimension in this case is $4+\phi^{3}$ and when intersected with the 80-dimensional Narain lattice one obtains the compactified Klein modular space. In all these circumstances, the golden mean which was recently discovered experimentally in a quantum system plays a central role [16]. The golden mean is the organizing centre for the Connes' dimensional function as well as the Jones' index. The irrationality of this most irrational number could be drawn into explaining the stability of elementary particles and the hierarchal problem (the desert hypothesis) as well as in explaining the Kirkwood gaps in the asteroid belt. 


\section{References}

[1] A. Connes, Noncommutative Geometry, Academic Press, New York, NY, USA, 1994.

[2] J. P. May, E-infinity Ring Spaces and E-Infinity Ring Spectra, Springer, Heidelberg, Germany, 1977.

[3] M. S. El Naschie, "A review of E-infinity theory and the mass spectrum of high energy particle physics," Chaos, Solitons and Fractals, vol. 19, no. 1, pp. 209-236, 2004.

[4] J. H. He, "A note on elementary cobordism and negative space," International Journal of Nonlinear Science and Numerical Simulations, vol. 11, no. 12, pp. 1093-1095, 2010.

[5] M. S. El Naschie, "Banach-tarski theorem and cantorian micro space-time," Chaos, Solitons and Fractals, vol. 5, no. 8, pp. 1503-1508, 1995.

[6] M. S. El Naschie, "Quantum collapse of wave interference pattern in the two-slit experiment: a set theoretical resolution," Nonlinear Science Letters A, vol. 2, no. 1, pp. 1-9, 2011.

[7] J. H. He, T. Zhong, L. Xu et al., "The importance of the empty set and noncomutative geometry in underpinning the foundations of quantum physics," Nonlinear Science Letters B, vol. 1, no. 1, pp. 15-24, 2011.

[8] M. S. El Naschie, "Superstrings, knots and noncommutative geometry in E-infinity space," International Journal of Theoretical Physics, vol. 37, no. 12, pp. 2935-2951, 1998.

[9] M. S. El Naschie, "Penrose universe and cantorian spacetime as a model for noncommutative quantum geometry," Chaos, Solitons and Fractals, vol. 9, no. 6, pp. 931-933, 1998.

[10] F. Büyükkılıç and D. Demirhan, "Cumulative diminuations with Fibonacci approach, golden section and physics," International Journal of Theoretical Physics, vol. 47, no. 3, pp. 606616, 2008.

[11] V. Jones and V. S. Sunder, Introduction to Subfactors, University Press, Cambridge, UK, 1997.

[12] L. Marek-Crnjac, "E-infinity Cantorian space-time from subfactors and knot theory," Chaos, Solitons and Fractals, vol. 32, no. 3, pp. 916-919, 2007.

[13] L. Marek-Crnjac, "On a connection between the limit set of the Möbius-Klein transformation, periodic continued fractions, El Naschie's topological theory of high energy particle physics and the possibility of a new axion-like particle," Chaos, Solitons and Fractals, vol. 21, no. 1, pp. 9-19, 2004.

[14] M. C. Gutzwiller, Chaos in Classical and Quantum Mechanics, Springer, New York, NY, USA, 1990.

[15] L. Marek-Crnjac, "The mass spectrum of high energy particles via El Naschie's E-infinity golden mean nested oscillators, the Dunkerly-Southwell eigenvalue theorems and KAM," Chaos, Solitons and Fractals, vol. 18, no. 1, pp. 125-133, 2003.

[16] R. Coldea, D. A. Tennant, E. M. Wheeler et al., "Golden ratio discovered in a quantum word," Science, vol. 8, no. 32, Article ID 5962, pp. 177-180, 2010. 

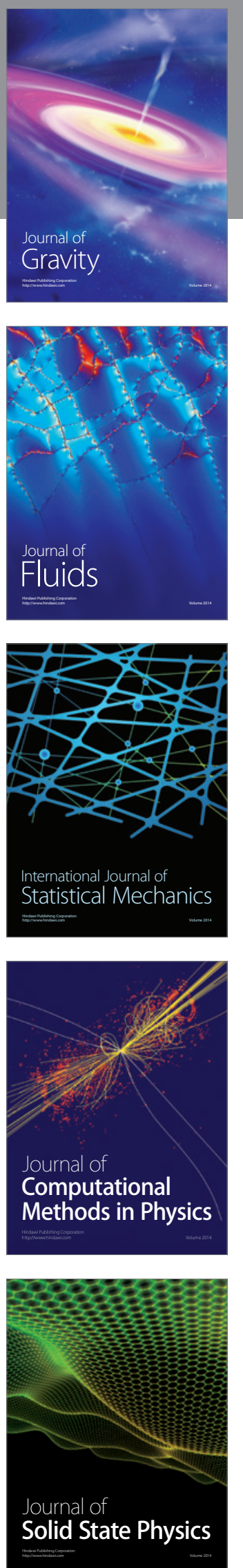

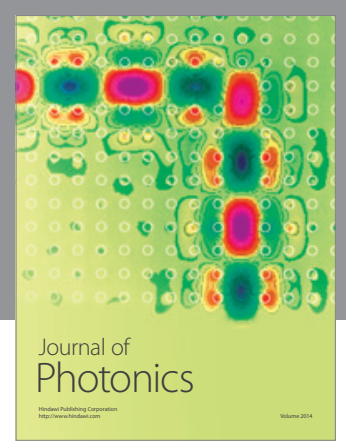

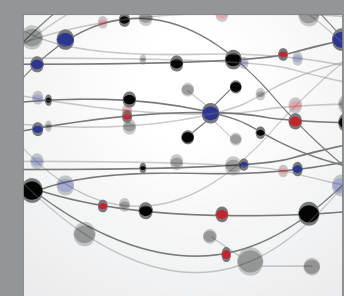

The Scientific World Journal
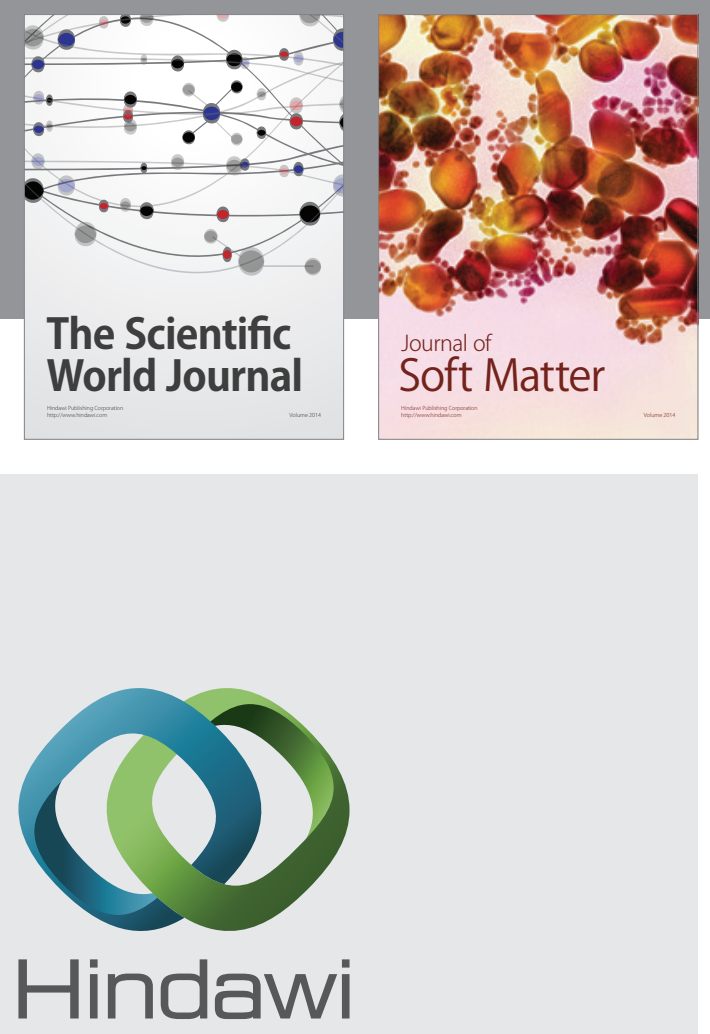

Submit your manuscripts at

http://www.hindawi.com
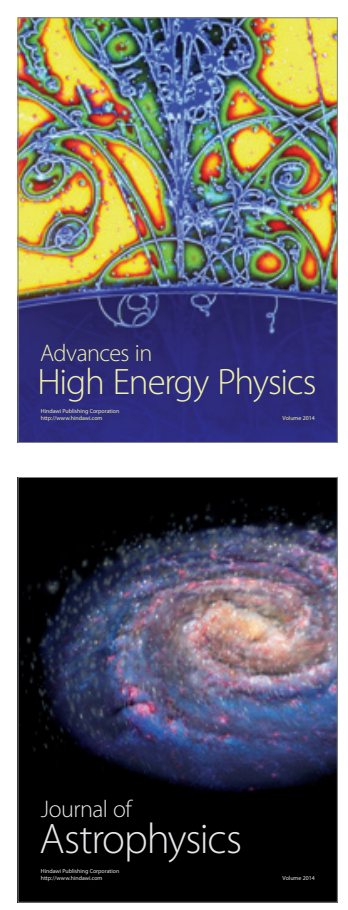
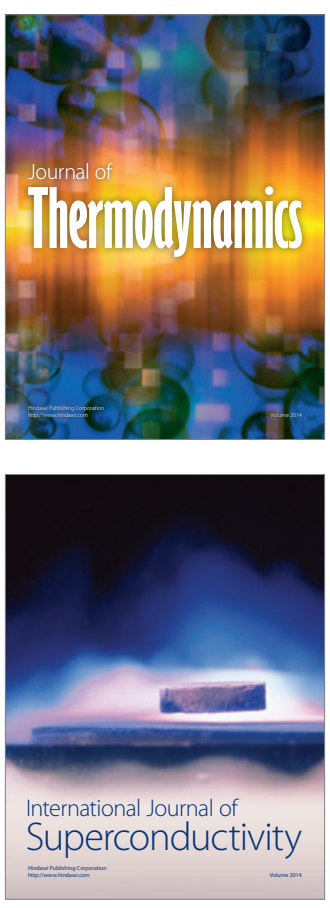
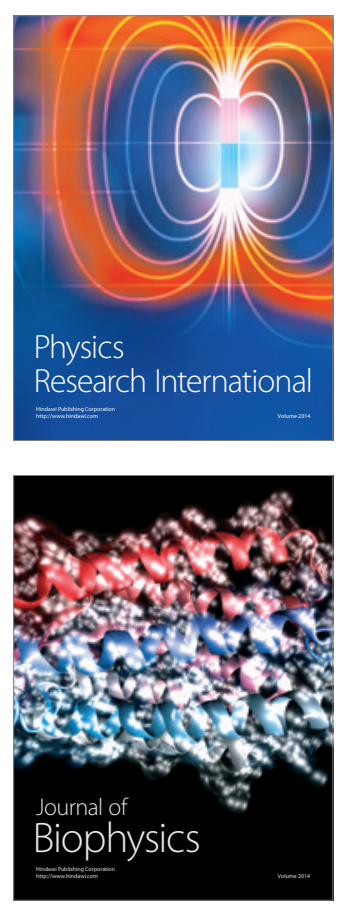
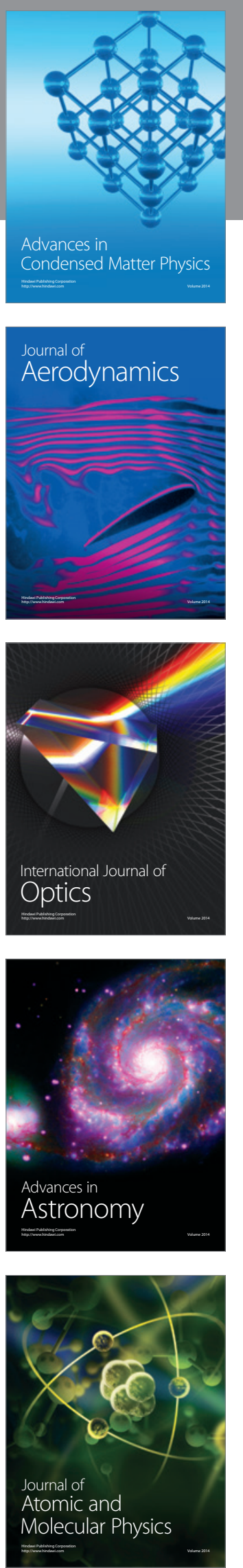